\title{
Short Lyapunov time: a method for identifying confined chaos
}

\author{
O. C. Winter, D. C. Mourão, and S. M. Giuliatti Winter
}

\begin{abstract}
UNESP - Univ. Estadual Paulista, Grupo de Dinâmica Orbital \& Planetologia, Guaratinguetá, CEP 12516-410, SP, Brazil e-mail: ocwinter@pq.cnpq.br, decmou@yahoo.com.br, silvia@feg.unesp.br
\end{abstract}

Received 19 June 2009 / Accepted 22 June 2010

\begin{abstract}
Context. The orbital instability of minor solar system bodies (asteroids, small satellites, moonlets, and particles) is frequently studied in terms of the Lyapunov characteristic exponent (LCE). Asteroids interior to Jupiter often exihibit very short Lyapunov times, $T_{\mathrm{L}}$, and very large radial variations, becoming Jupiter's crossers and escapers. However, a few cases of asteroids with very short $T_{\mathrm{L}}$ and no significant radial variation have been found. These orbits were called "confined chaos" or even "stable chaos". This feature also appeared in the case of moonlets embedded in Saturn's F ring and disturbed by the nearby satellites Prometheus and Pandora.

Aims. We present a simple approach to estimating the contribution of the radial component of the LCE to identify trajectories in the "confined chaos" regime.

Methods. To estimate the radial contribution to the maximum LCE, we considered a rotating reference system in which one of the axis was aligned with the radial direction of the reference trajectory. Measuring the distance in the phase space between the two nearby orbits then allowed us to separate the contribution of the radial component from the others. We applied the method to two different dynamical systems: (a) an asteroid around the Sun disturbed by Jupiter; (b) a moonlet of Saturn's F-ring disturbed by the satellites Prometheus and Pandora.

Results. In all cases, we found that the method of comparing the radial contribution of the LCE to the entire contribution allows us to correctly distinguish between confined chaos and escapers.
\end{abstract}

Key words. chaos - methods: numerical - celestial mechanics

\section{Introduction}

The orbital instability of minor solar system bodies (asteroids, small satellites, moonlets, and particles) is frequently studied in terms of the Lyapunov characteristic exponent (LCE). There are many cases where a short Lyapunov time, $T_{\mathrm{L}}$, is associated with the body moving on a trajectory that crosses the orbit of a large perturber, i.e, a trajectory that exhibits a large radial variation. The time required for that to happen is called the "event time" $\left(T_{\mathrm{E}}\right)$. Several studies involving the instability of asteroids disturbed by Jupiter and/or other planets (Soper et al. 1990; Lecar et al. 1992; Franklin et al. 1993; Levison \& Duncan 1993; Murison et al. 1994; Whipple 1995) have claimed that there is a simple relation between the Lyapunov time, $T_{\mathrm{L}}$, and the event time, $T_{\mathrm{E}}$ (to become a Jupiter's crosser, for example) given by

$\log \left(T_{\mathrm{E}} / T_{0}\right)=a+b \log \left(T_{\mathrm{L}} / T_{0}\right)$,

where $T_{0}$ is an appropriate normalizing period, $a$ and $b$ are constants empirically determined. Urminsky and Heggie (2009) used the Poincaré surface of section of the three-body problem to refine the values of these constants.

However, Milani and coworkers (Milani \& Nobili 1992; Milani et al. 1997) argued against the idea of this simple relation by drawing attention to the discovery of asteroids with short $T_{\mathrm{L}}$ that do not become planet crossers, nor exhibit any large radial variations, for a thousand times the $T_{\mathrm{L}}$. They referred to this bahaviour as "stable chaos".

These two conflicting results, as discussed by Morbidelli \& Froeschlé (1996), correspond to two different dynamical regimes. In the regime called the "Nekhoroshev regime", the relationship between $T_{\mathrm{E}}$ and $T_{\mathrm{L}}$ must be exponential $\left(T_{\mathrm{E}} \sim \exp \left(T_{\mathrm{L}}\right)\right)$, while in the second regime, the "resonant overlapping regime", the relationship between $T_{\mathrm{E}}$ and $T_{\mathrm{L}}$ is polynomial $\left(T_{\mathrm{E}} \sim T_{\mathrm{L}}^{\beta}, \beta\right.$ is a positive constant). Guzzo \& Morbidelli (1997) and Morbidelli \& Guzzo (1997) demonstrated that the Nekhoroshev stability can be applied to describe the complicated problem of the motion of an asteroid. The asteroids that are on chaotic orbits but have motions that are stable over a long time are in the Nekhoroshev stability regime. The Nekhoroshev theorem can be applied to analyse the system because the masses of the planets with respect to the Sun, in addition to their eccentricities and inclinations, are all small parameters. However, this method cannot be applied to asteroids located inside or close to some low-order mean motion resonances.

Guzzo et al. (2002) analysed the long-term stability of a sample of real asteroids using the Fourier analysis of chaotic motions (Guzzo \& Benettin 2001). They found that some of these asteroids are in the Nekhoroshev stability regime. To verify whether the method can distinguish between the asteroid trajectory types they analysed a sample of asteroids located in different regions of the main belt and verified that it is possible to identify the asteroids in different dynamical regimes, such as those described by the Nekhoroshev stability or those described by the KAM theory.

Winter et al. (2007) studied the orbital evolution of moonlets embedded in Saturns' F ring, disturbed by the shepherd satellites, Prometheus and Pandora. They found that despite having very short Lyapunov times, the majority of the moonlets do not cross the orbits of the nearby satellites. They concluded that the moonlets exhibit a confined chaotic behaviour.

Hence, the problem is "how do we identify whether a short $T_{\mathrm{L}}$ implies a confined chaos or a escaper?". The LCE infers the overal maximum exponent value. It does not provide the radial 
contribution to this value. In the present work, we propose a simple approach to estimate the contribution of the radial component of the Lyapunov characteristic exponent. The computation is performed simultaneously with that of the LCE. If the radial component and the LCE are of the same order it implies that we have a case of escaper, but if the radial component contribution is orders of magnitude smaller than the LCE we have a case of confined chaos.

In the next section, we describe the methodology for the computation of the radial contribution of the LCE. In Sect. 3 we study the case of asteroids disturbed by Jupiter and the case of moonlets in the F ring disturbed by the satellites Prometheus and Pandora. In the last section, we present our final comments.

\section{Methodology}

The Lyapunov characteristic exponent (LCE) is a tool for measuring the rate of divergence of two nearby trajectories in the phase space. According to Benettin et al. (1980), it can be computed by performing the numerical integration of the equations of motion of two nearby trajectories, where its maximum value is given by

$\gamma=\lim _{k \rightarrow \infty} \frac{1}{k \tau} \sum_{i=0}^{k} \ln \frac{d(i \tau)}{d_{0}}$,

and $d(i \tau)$ is the distance in the phase space between the two trajectories (a reference trajectory and a shadow one) initially separated by a distance $d_{0}$. The computation is made at a fixed time interval $\tau$, after which the separation vector of the nearby trajectory is rescaled to maintain measurements of the local divergence of the two orbits. The system is said to be regular if $\gamma=0$ and chaotic if $\gamma>0$.

The inverse of the LCE is known as Lyapunov time $\left(T_{\mathrm{L}}=\right.$ $1 / \gamma)$. In the case of a large LCE, we have a short $T_{\mathrm{L}}$, which implies that the system is "highly" chaotic, i.e., the two nearby trajectories diverge exponentially very quickly.

In this section, we consider a 2-dimensional system, but it could be easily expanded to the 3 -dimensional case. In a cartesian coordinate system $O X Y$, a body is identified in the phase space, at a given time, by the coordinates of its position $(X, Y)$ and velocity $\left(V_{X}, V_{Y}\right)$. Defining the subscript 1 to refer to the reference trajectory and the subscript 2 to the shadow trajectory, the distance in the phase space between the two trajectories is given by

$$
\begin{aligned}
d= & {\left[\left(X_{1}-X_{2}\right)^{2}+\left(Y_{1}-Y_{2}\right)^{2}\right.} \\
& \left.+\left(V_{X 1}-V_{X 2}\right)^{2}+\left(V_{Y 1}-V_{Y 2}\right)^{2}\right]^{1 / 2}
\end{aligned}
$$

In the computation of the LCE (Eq. (2)), the value of $d$ is measured periodically at a fixed time interval $\tau$, during $k$ interactions. Ideally, $k$ should tend to infinity. However, in practice, the value of $\gamma$ is computed by evaluating

$\gamma=\frac{1}{k \tau} \sum_{i=0}^{k} \ln \frac{d(i \tau)}{d_{0}}$,

which monitors the evolution of its value with increasing values of $k$.

To estimate the contribution of the radial component of $\gamma$, we compute the distance, $d$, in the phase space using a rotating cartesian coordinate system $\mathrm{O} \xi \eta$, which keeps the reference

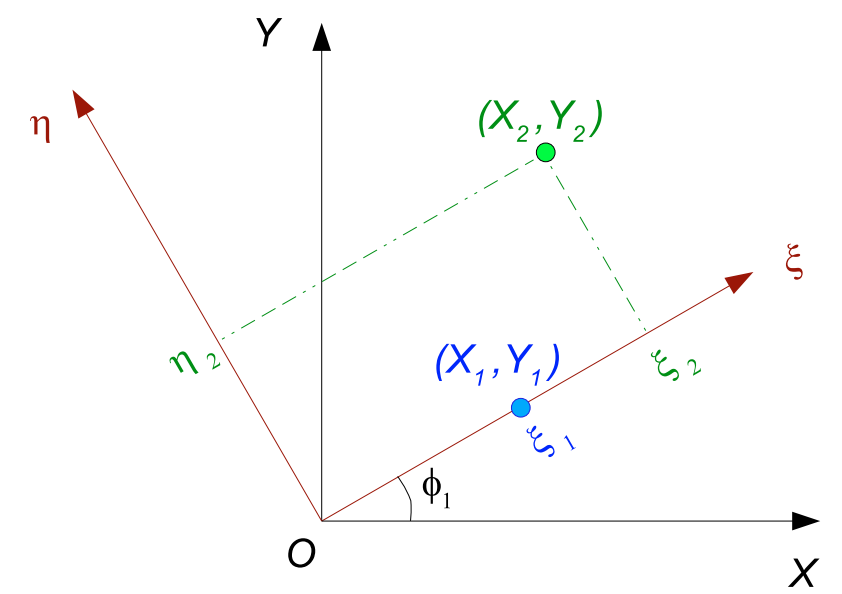

Fig. 1. Cartesian coordinate systems. The inertial coordinate system is represented by OXY. The rotating reference frame is represented by $\mathrm{O} \xi \eta$. The angle of rotation is given by $\phi_{1}=\arctan \left(Y_{1} / X_{1}\right)$. Note that the $\xi$ axis is always in the radial direction of the reference body (subscript 1).

body over the $\xi$ axis (Fig. 1). We note that the $\xi$ axis is always in the radial direction of the motion of the reference body.

In the rotating coordinate system, a body is identified in the phase space, at a given time, by the coordinates of its position $(\xi, \eta)$ and its velocity $\left(V_{\xi}, V_{\eta}\right)$. Then, the rotation matrix,

$\mathbf{M}=\left(\begin{array}{rr}\cos \phi_{1} & \sin \phi_{1} \\ -\sin \phi_{1} & \cos \phi_{1}\end{array}\right)$,

where $\phi_{1}=\arctan \left(Y_{1} / X_{1}\right)$, is used to convert the inertial coordinates into the rotated coordinates for both, the reference trajectory and the shadow trajectory, by calculating

$\left(\begin{array}{c}\xi_{i} \\ \eta_{i}\end{array}\right)=\mathbf{M}\left(\begin{array}{c}X_{i} \\ Y_{i}\end{array}\right)$

and

$\left(\begin{array}{c}V_{\xi i} \\ V_{\eta i}\end{array}\right)=\mathbf{M}\left(\begin{array}{c}V_{X i} \\ V_{Y i}\end{array}\right)$

for $i=1,2$. The distance in the phase space between the two trajectories is then given by

$\begin{aligned} d= & {\left[\left(\xi_{1}-\xi_{2}\right)^{2}+\left(\eta_{1}-\eta_{2}\right)^{2}\right.} \\ & \left.+\left(V_{\xi 1}-V_{\xi 2}\right)^{2}+\left(V_{\eta 1}-V_{\eta 2}\right)^{2}\right]^{1 / 2} .\end{aligned}$

We now propose to compute the distance between the trajectories considering only the radial component (the term in the first parenthesis of Eq. (8)). From Eq. (4), we then find

$\gamma_{\mathrm{rad}}=\frac{1}{k \tau} \sum_{i=0}^{k} \ln \frac{\left|\xi_{1}-\xi_{2}\right|}{d_{0}}$

which is the contribution of the radial component to the LCE. We can also compute the LCE without the radial component contribution to be

$\gamma_{*}=\frac{1}{k \tau} \sum_{i=0}^{k} \ln \frac{\sqrt{\left(\eta_{1}-\eta_{2}\right)^{2}+\left(V_{\xi 1}-V_{\xi 2}\right)^{2}+\left(V_{\eta 1}-V_{\eta 2}\right)^{2}}}{d_{0}}$.

Finally, for an orbital system with a short Lyapunov time, one can determine whether $\gamma$ and $\gamma_{\text {rad }}$ are of the same order 
O. C. Winter et al.: Planet crossers or confined chaos?
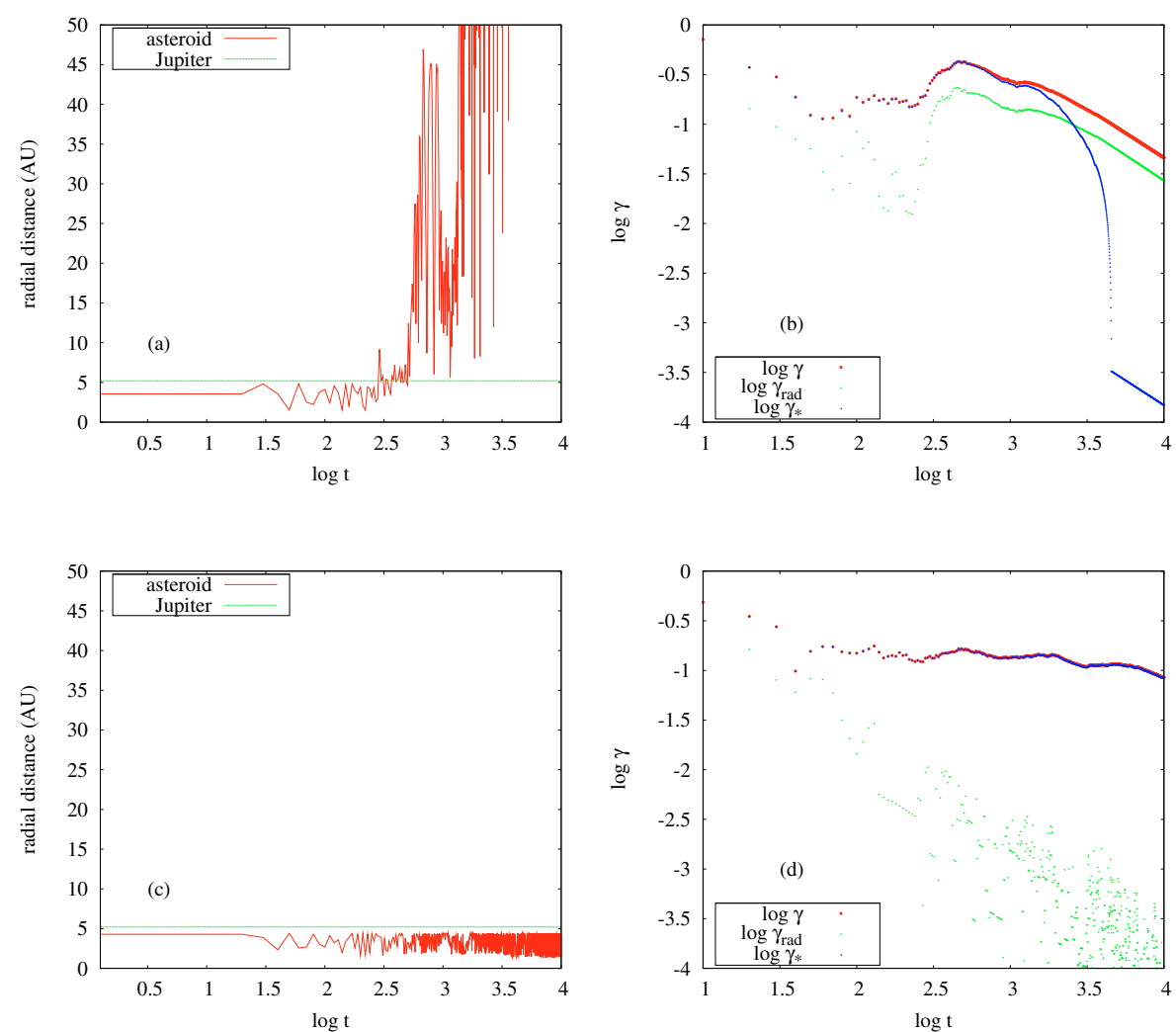

Fig. 2. Results of the simulations for two asteroids disturbed by Jupiter: Plots a)-b) are for an example of an "escaper"; Plots c)-d) are for an example of "confined chaos". The plots in the first column show the temporal evolution of the orbital radius of each asteroid. The green line indicates the orbital radius of Jupiter. Plots in the second column show the Lyapunov characteristic exponent of the each asteroid. Three computations are shown: (1) the complete LCE, given by $\gamma$ (in red); (2) the contribution of the radial component, given by $\gamma_{\text {rad }}$ (in green); and (3) the LCE without the radial contribution, given by $\gamma_{*}$ (in blue).

or $\gamma_{\mathrm{rad}} \ll \gamma$. We can then say whether the trajectory is expected to show a significant radial variation or not. On the light of the Nekhoroshev theorem, an orbital system with a short Lyapunov time, whose computations show $\gamma_{\mathrm{rad}} \ll \gamma$, is in the Nekhoroshev-like regime of effective stability.

\section{Confined chaos or escaper?}

We tested the approach described in the previous section on two different dynamical systems. We studied the case of an asteroid disturbed by Jupiter and the case of a moonlet of the $\mathrm{F}$ ring disturbed by the satellites Prometheus and Pandora. The systems were numerically simulated using a Burlish-Stoer integrator. They were integrated for $10^{4}$ orbital periods of the perturber. In the case of the asteroid, we assumed the orbital period of Jupiter. In the case of the moonlet, we used an orbital period given by the mean value between the orbital periods of Prometheus and Pandora.

\subsection{Asteroid disturbed by Jupiter}

Firstly we analyse the case of an asteroid orbiting the Sun and disturbed by Jupiter, in the framework of the planar circular restricted three-body problem. We adopted the mass and semimajor axis of Jupiter given in Murray and Dermott (1999). The asteroids were initially placed on the line between Sun and Jupiter, in circular orbit with semi-major axis $2.9 \mathrm{AU} \leq a \leq$ 3.8 AU, where $\Delta a=0.01 \mathrm{AU}$.
In Fig. 2a, we present the temporal evolution of the orbital radius of one asteroid in a "escaper" regime. The unit of distance in this plot is normalized such that the initial orbital radius of the asteroid is equal to one. The green line indicates the orbital radius of Jupiter, the perturber. This plot reveals a very large amplitude of variation in the orbital radius of the asteroid. The orbital radius of the asteroid increases more than 250 times from its initial value. The results from the study of the LCE for this asteroid are plotted in Fig. 2b. In the plot, we show $\gamma$ (red), $\gamma_{\text {rad }}$ (green), and $\gamma_{*}$ (blue) versus time on a log-log scale. This figure clearly shows that the evolution of $\gamma_{\text {rad }}$ closely follows the evolution of $\gamma$. Therefore, the contribution of the radial component to the LCE is significant, not negligible, along the whole length of integration. This is compatible with the large variation in the orbital radius (Fig. 2a).

In Fig. 2c, we present the temporal evolution of the orbital radius of one asteroid in a "confined chaos" regime. This plot reveals a very small amplitude of variation in the orbital radius of the asteroid. The results from the study of the LCE for this asteroid are plotted in Fig. 2d. This figure clearly shows that, as the system evolves, the values of $\gamma_{\mathrm{rad}}$ are orders of magnitude smaller than the values of $\gamma$. Therefore, the contribution of the radial component to the LCE can be considered to be negligible. This is compatible with the very small variation in the orbital radius of this asteroid (Fig. 2c).

We performed the simulation of a sample of 901 hypothetical asteroids. About $54 \%$ of them presented short Lyapunov time (of just a few hundred years). From those, about $75 \%$ exhibited a significant variation in the orbital radius, such that they became escapers. We found that the contribution of the radial component 


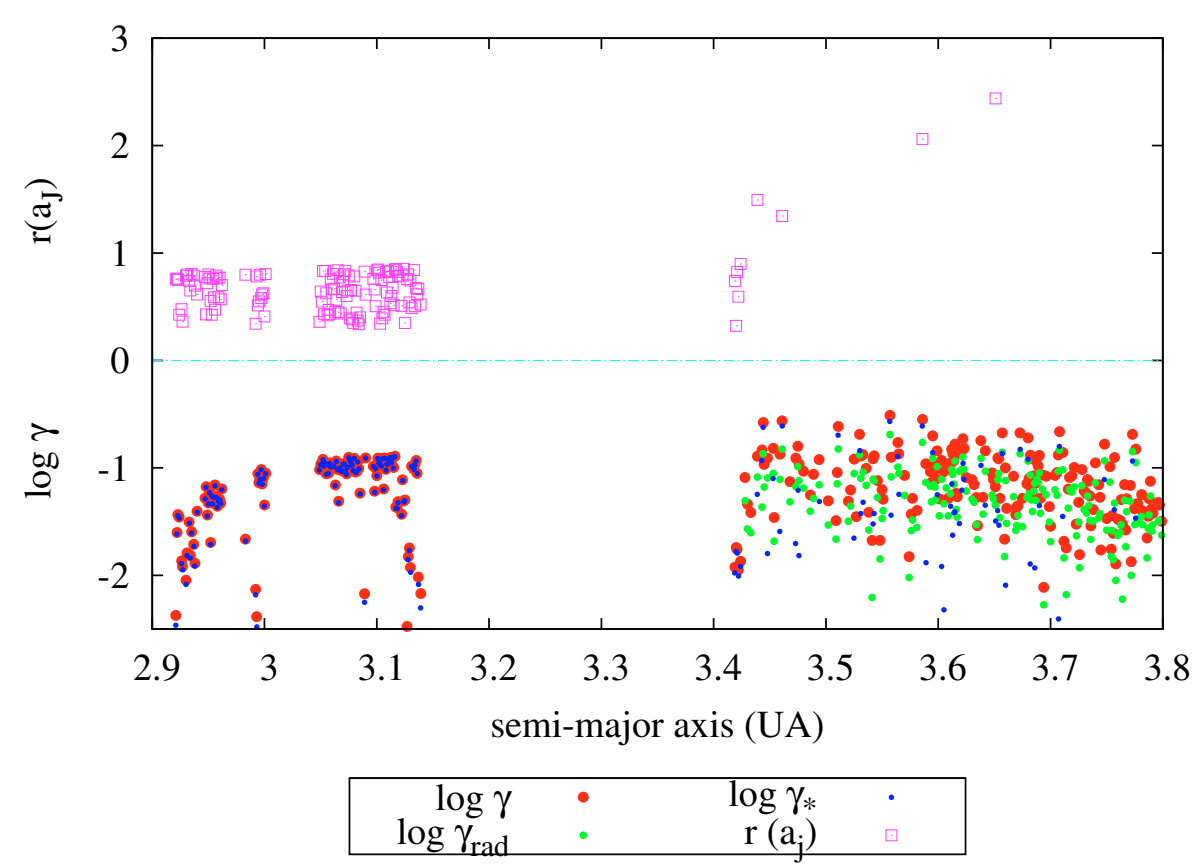

Fig. 3. Overview of the results for the sample of simulations for asteroids disturbed by Jupiter. In this plot, we consider only the data concerning the asteroids with short Lyapunov time (of just a few hundred years). This figure is divided by the light blue line into two parts, in the vertical axis: (1) positive values indicate the final orbital radius of the asteroid (pink), in units of Jupiter's orbital radius, and (2) negative values indicate the logarithm of Lyapunov characteristic exponent of the asteroid, where (i) the complete LCE, given by $\gamma$, is in red; (ii) the contribution of the radial component, given by $\gamma_{\mathrm{rad}}$, is in green; and (iii) the LCE without the radial contribution, given by $\gamma_{*}$, is in blue. The horizontal axis indicates the initial semi-major axis of the asteroid.

Table 1. Orbital initial conditions and masses of the satellites.

\begin{tabular}{cccccc}
\hline \hline & $a(\mathrm{~km})$ & $e$ & $\omega\left(^{\circ}\right)$ & $f\left({ }^{\circ}\right)$ & Mass $\left(M_{\text {Saturn }}\right)$ \\
Prometheus & 139377.33 & 0.00192 & 228 & 188.526 & $5.8 \times 10^{-10}$ \\
Pandora & 140712.63 & 0.00450 & 57 & 25.120 & $3.43 \times 10^{-10}$ \\
\hline
\end{tabular}

to the LCE for all these planet crossers are comparable to the total value of the LCE. The remaining $25 \%$ did not even became Jupiter's crossers and their $\gamma_{\text {rad }}$ value were all found to be negligible compared to their $\gamma$ values. Our approach therefore worked very well in all these cases. In Fig. 3 we present an overview of these results. The asteroids that do not have a $\gamma_{\text {rad }}$ value (green) close to their $\gamma$ value (red) are in the "confined chaos" regime, exhibiting a last orbital radius value (pink) smaller than that of Jupiter. On the other hand, the asteroids that do have a $\gamma_{\text {rad }}$ value (green) close to their $\gamma$ value (red) are in the "escapers" regime. Their last orbital radius value (pink) is many times larger than that of Jupiter. Consequently, most of them do not appear in the plot.

\subsection{Moonlet disturbed by Prometheus and Pandora}

The second dynamical system that we consider is the motion of moonlets located in the region of the F ring of Saturn, disturbed by the close satellites Prometheus and Pandora. This dynamical system is assumed to be planar and the moonlet is of negligible mass. The gravitational potential of Saturn takes into account its harmonics $J_{2}, J_{4}$, and $J_{6}$, given by Evans (2001). We performed simulations for 396 hypothetical moonlets of the F ring distributed within the following initial orbital elements: semimajor axis, $a=a_{F} \pm \delta a$ with $\delta a=0,10,20,30,40,50 \mathrm{~km}$, where $a_{F}=140,050 \mathrm{~km}$ is the mean value for the F ring; eccentricity, $e=0.00260$; true anomaly, $0^{\circ} \leq f \leq 360^{\circ}$ with $\Delta f=10^{\circ}$; and argument of the pericentre, $\omega=0^{\circ}$. The initial orbital elements and masses of the satellites Prometheus and Pandora are given in Table 1.

The simulations for the whole sample of moonlets inferred short Lyapunov time (shorter than two hundred orbital periods). Using our approach, we verified that in none of the simulations is the radial contribution of the Lyapunov exponent, $\gamma_{\text {rad }}$, comparable to $\gamma$. We did not find only trajectories that did not cross the orbits of any of the satellites. We also found many trajectories that did cross the orbit of Prometheus or Pandora, and some that crossed both orbits. However, all of them remained in trajectories confined to a region close to the satellites.

In Fig. 4, we present representative behaviours detected in our simulations. In all figures, the plots in the first column present the temporal evolution of the orbital radius of a moonlet, where the unit of distance is normalized such that the mean orbital radius of the $\mathrm{F}$ ring, $a_{\mathrm{F}}$, is equal to one. The green and blue lines indicate the orbital radius of the satellites Prometheus and Pandora, respectively. In Fig. 4, the plots in the second column present the results from the study of the LCE. In these plots, we show $\gamma$ (red), $\gamma_{\text {rad }}$ (green) and $\gamma_{*}$ (blue) versus time in a log-log scale. In the example A (Fig. 4a and b), the plot in Fig. 4a reveals a small amplitude of variation in the orbital radius of the moonlet. The moonlet remains confined between the two close satellites. This agrees, as ilustrated in Fig. 4b, with the negligible contribution of $\gamma_{\mathrm{rad}}\left(<10^{-10}\right)$ in comparison to the total Lyapunov exponent, $\gamma\left(>10^{-2}\right)$. The green dots, associated with $\gamma_{\text {rad }}$, do not appear in the plot because their values are several orders of magnitude smaller than the lower limit of the vertical axis. Hence, the contribution of the radial component to the LCE is completely negligible. We note also that the evolution of $\gamma_{*}$ closely follows the evolution of $\gamma$, in a similar way to the evolution found for the asteroid shown in Fig. 2c, d.

In the example B (Fig. 4c, d), the plot in Fig. 4c reveals a small amplitude of variation for the orbital radius of the moonlet. However, this variation is large enough to cross the orbit of Pandora, despite the results of the evolution for $\gamma_{\mathrm{rad}}, \gamma_{*}$, and $\gamma$, shown in Fig. 4d, being almost identical to those of the previous example, shown in Fig. 4b. 
O. C. Winter et al.: Planet crossers or confined chaos?
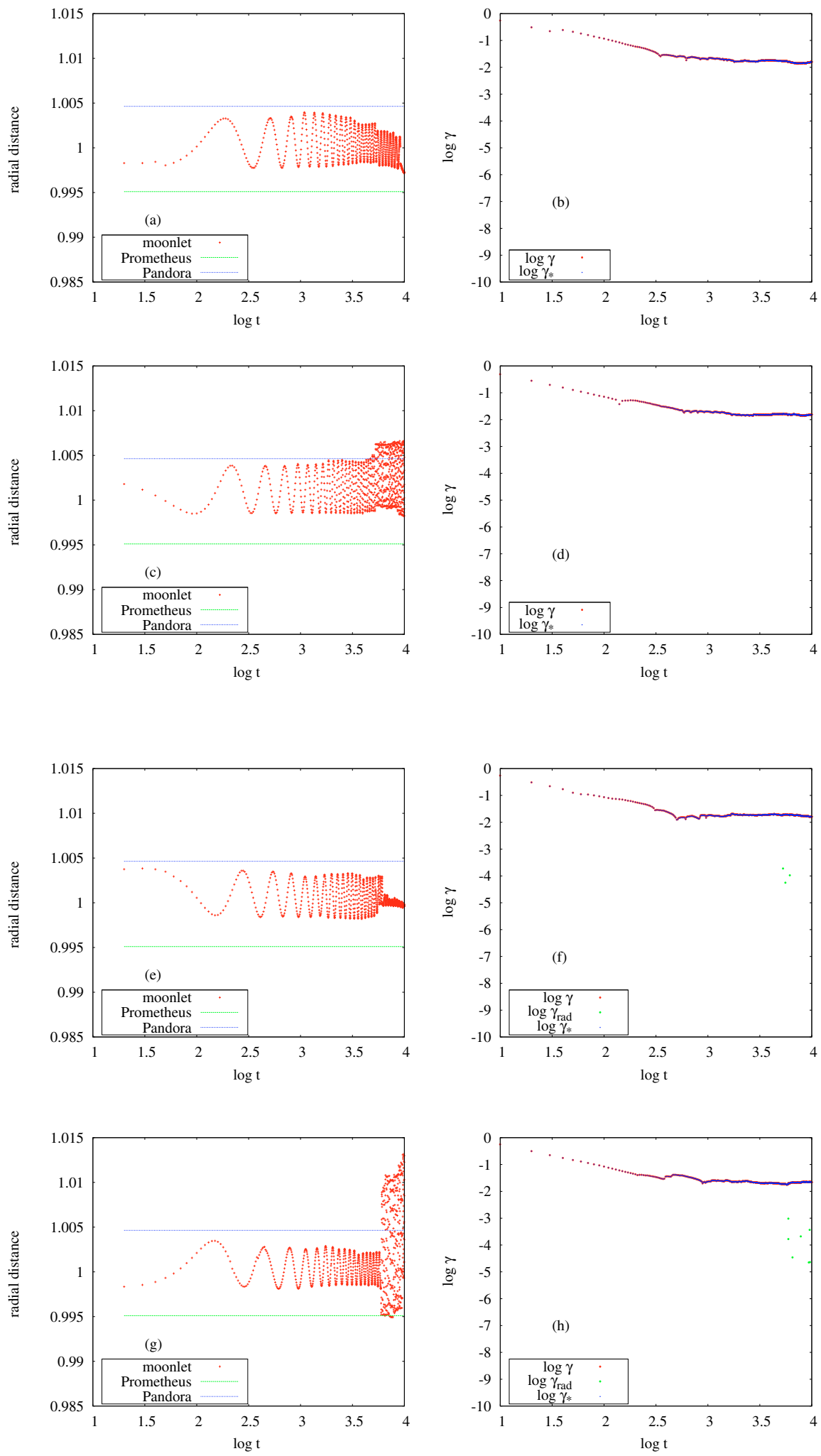

Fig. 4. Results for four moonlets disturbed by the satellites Prometheus and Pandora, examples A, B, C, and D, from the first to the fourth lines, respectively. The plots in the first column show the temporal evolution in the orbital radius of each moonlet. The unit of distance in these plots is normalized such that the mean orbital radius of the F ring is equal to one. The green and blue lines indicate the mean orbital radius of the satellites Prometheus and Pandora, respectively. The plots in the second column show the Lyapunov characteristic exponent of each moonlet. Three computations are shown: (1) the complete LCE, given by $\gamma$ (in red); (2) the contribution of the radial component, given by $\gamma_{\text {rad }}$ (in green); (3) the LCE without the radial contribution, given by $\gamma_{*}$ (in blue). In the examples A and B, the green dots, associated with $\gamma_{\text {rad }}$, do not appear in the plot because their values are several orders of magnitude smaller than the lower limit of the vertical axis. 
In the examples $\mathrm{C}$ and $\mathrm{D}$, shown in Figs. $4 \mathrm{e}, \mathrm{f}$ and $4 \mathrm{~g}$, $\mathrm{h}$, we find that one moonlet remains confined between the two satellites and the other crosses the orbits of Prometheus and Pandora. In both cases, the evolution of $\gamma_{*}$ closely follows the evolution of $\gamma$, as in the examples A and B. The only difference now is that there are a few green dots in the plots of the LCE. These few green dots do not change the conclusion that the contribution of the radial component to the LCE, $\gamma_{\mathrm{rad}}$, is negligible compared to $\gamma$.

Therefore, the results of the simulations for this system demonstrated that none of the trajectories exhibited a significant radial variation, although many of them crossed the orbit of at least one of the perturbing satellites. This seems to be a natural outcome, because the disturbers are very small bodies $\left(10^{-10}\right.$ of the central body mass) and their orbits are very close to the initial moonlets' orbits. Thus, moonlets might frequently cross the orbits of Prometheus and/or Pandora without experiencing a strong perturbation from these satellites. Records of our simulations show that all the moonlets studied remain confined in a radial range between $136,408 \mathrm{~km}$ and $145,091 \mathrm{~km}$. This means that at most, the moonlets go to within only $1.94 \%$ of Prometheus pericenter and only $2.65 \%$ outside Pandora's apocentre.

\section{Final comments}

A common problem in the dynamics of minor solar system bodies are the gravitational perturbations caused by larger bodies, as in the case of asteroids disturbed by Jupiter, or ring moonlets disturbed by nearby satellites. One of the most useful tools for detecting whether the perturbation introduces chaotic behaviour into the dynamics of the minor body is the Lyapunov characteristic exponent.

In the cases when short Lyapunov times are found, it would be natural to expect large radial variations, with the body crossing the perturber's orbit and escaping. However, this is not always the case. We found asteroids with short $T_{\mathrm{L}}$ that do not cross Jupiter's orbit, and moonlets of Saturn's F ring with short $T_{\mathrm{L}}$, that remained confined to region nearby the shepherd satellites.

A problem with using the maximum LCE in these cases is that it does not provide information about how much of it is contributed to the radial direction of motion.

In the present work, we have introduced a very simple approach to estimate the contribution of the radial variation in the Lyapunov characteristic exponent, $\gamma_{\mathrm{rad}}$. The computation is easy to implement and can be performed simultaneously with that of the LCE, $\gamma$.
We have tested our approach on two different dynamical systems. In the case of asteroids disturbed by Jupiter, a comparison between the evolution of the values of $\gamma_{\mathrm{rad}}$ and $\gamma$ has been shown to clearly indicate whether the orbit of the asteroid considered was a case of "confined chaos" or a case of an "escaper". The method worked very well for $100 \%$ of the simulations performed considering this system.

In the case of a moonlet disturbed by the satellites Prometheus and Pandora, all the trajectories were found to be in a "confined chaos" regime. This is because the disturbers are very small bodies $\left(10^{-10}\right.$ of the central body mass) with orbits very close to the moonlet, one on each side of its orbit. As a consequence, moonlets might frequently cross the orbits of Prometheus and/or Pandora without experiencing a strong perturbation from these satellites. Using our approach, we have verified that in none of the simulations is the radial contribution of the Lyapunov exponent, $\gamma_{\mathrm{rad}}$, comparable to $\gamma$. Therefore, our method for assessing the radial contribution of the LCE allows one to correctly distinguish between confined chaos and escapers regimes.

Acknowledgements. The authors would like to thank Tadashi Yokoyama and Ricardo Cordeiro for helpful comments and Zoran Knezevic for providing some useful references. The suggestions of an anonymous referee also improved this paper. This work was supported by CNPq and FAPESP.

\section{References}

Benettin, G., Galgani, L., Giorgilli, A., \& Strelcyn J.-M. 1980, Meccanica, 15, 9 Evans, M. 2001, PhD Thesis, Queen Mary College, Univ. London Franklin, F., Lecar, M., \& Murison, M. 1993, AJ, 105, 2336

Guzzo, M., \& Benettin, G. 2001, Discrete Cont. Dyn. Sys. Series B1 (1) Guzzo, M., \& Morbidelli, A. 1997, Cel. Mech. Dyn. Ast., 66, 255

Guzzo, M., Knezevic, Z., \& Milani, A. 2002, Cel. Mech. Dyn. Ast., 83, 121 Lecar, M., Franklin, F., \& Murison, M. 1992, AJ, 104, 1230

Levison, H. F., \& Duncan, M. J. 1993, ApJ, 406, L35

Milani, A., \& Nobili, A. M. 1992, Nature, 157, 569

Milani, A., Nobili, A. M., \& Knezevic, Z. 1997, Icarus, 125, 13

Morbidelli, A., \& Froeschlé, C. 1996, Cel. Mech. Dyn. Ast., 63, 227

Morbidelli, A., \& Guzzo, M. 1997, Cel. Mech. Dyn. Ast., 65, 107

Murison, M., Lecar, M., \& Franklin, F. 1994, AJ, 108, 2323

Murray, C. D., \& Dermott, S. F. 1999, Solar System Dynamics (Cambridge: Cambridge University Press)

Soper, P., Franklin, F., \& Lecar, M. 1990. Icarus, 87, 265

Urminsky, D. J., \& Heggie, D. C. 2009, MNRAS, 392, 1051

Whipple, A. L. 1995, Icarus, 115, 347

Winter, O. C., Mourão, D. C., Giuliatti Winter, S. M., Spahn, F., \& da Cruz, C. 2007, MNRAS, 380, L54 